\title{
Parameters sensitivity analysis in a solenoid common-rail injector model
}

\author{
J.L. Perona-Navarro ${ }^{1}$, A. Palomar-Torres ${ }^{1}$, E. Torres-Jiménez ${ }^{1}$, O. Armas ${ }^{2}$, L. Lešnik ${ }^{3}$, \\ F. Cruz-Peragón ${ }^{1}$ \\ ${ }^{1}$ Department of Mechanical and Mining Engineering \\ E.P.S. of Jaén, University of Jaén \\ Campus Las Lagunillas s/n, 23071 Jaén (Spain)
}

Phone number: +34 953212367, e-mail: ilpn0001@ red.ujaen.es, apt00010@ red.ujaen.es, etorres@ujaen.es, fcruz@ujaen.es

\begin{abstract}
${ }^{2}$ Escuela de Ingeniería Industrial y Aeroespacial, Universidad de Castilla La Mancha Campus Real Fábrica de Armas, Av. Carlos III s/n, Edif. Sabatini, 45071, Toledo (Spain) Phone number: +34 925 268000, e-mail: Octavio.Armas@ uclm.es
\end{abstract}

\author{
${ }^{3}$ Institute of Energy, Process and Environmental Engineering, Faculty of Mechanical Engineering, \\ University of Maribor, Smetanova 17, SI-2000 Maribor (Slovenia) \\ Phone number: +386 2220 7734, e-mail: $\underline{\text { luka.lesnik@um.si }}$
}

\begin{abstract}
Parameter identification techniques are applied to find suitable values of unknown key input factors into a mathematical model. Usually, these factors are determined through optimization algorithms. In this case, the establishment of consistent initial values, and their corresponding searching ranges (which are unknown), is quite important to provide reliable results after the identification process. In this work, a sensitivity analysis is carried out to evaluate the influence of the main parameters involved in a numerical model used to simulate a common-rail solenoid injector. Experimental measurements for several operating conditions were done, and their results were compared with the model outputs to establish an objective function from which to perform the assessment. As a result of this study, it was established the importance of each parameter included in the model: firstly, those parameters with low variability were selected to be removed from the identification procedure, assuming a value for them that do not affect the model results; finally, the rest of parameters remained in the algorithm. After applying the aforementioned identification process, some accurate results are provided and discussed in order to propose improvements for future works.
\end{abstract}

Key words. Identification algorithm, Sensitivity analysis, Fuel injection system, Mathematical modelling, Diesel fuel.

\section{Introduction}

The validation of a mathematical model implies that the results of the model (outputs) match the experimental results. For this purpose, it is essential to know the value of the model input parameters. Nevertheless, some of them are unknown and, once the experimental results are obtained, it is possible to identify them.
Generally, the identification process uses optimization algorithms, both conventional (based on Newton method) [1] or intelligent (e.g. Genetic Algorithms (GA)) [2] These algorithms search for the minimum value of a defined objective function $\Psi$, which is an indicator of the approach goodness between the actual (experimental measurements) and the simulated output parameter of the system. The application of this methodology is very common in the field of combustion engines [3].

To successfully accomplish this identification, it is necessary to evaluate the influence of each parameter on the model results: for those unknown parameters whose variation do not significantly change the simulated results a fix value can be assumed, removing them from the optimization algorithm, which simplifies the identification process. On the other hand, the rest of unknown parameters must be carefully evaluated, by setting an adequate searching range for determining their actual values. If these searching ranges are stablished in a very wide interval or bad located, they could lead to physically of geometrically incompatible results. Sensitivity analysis plays a fundamental role in order to appropriately chose these initial settings: it is a useful tool to evaluate the influence of the input parameter values variation on the model outputs (evaluated using the objective function) [4].

This paper summarizes the work done regarding the sensitivity analysis for a set of input parameters that are required to define a solenoid common-rail injector model. Finally, a preliminary identification process for the evaluation of the goodness of the initial set of ranges selected for the optimization algorithm is performed and the main results discussed. 
The interest of this work is that represents an initial step of a systematic procedure for tuning a model. Among its advantages are minimizing the number of parameters required for the subsequent calibration process and setting the search direction to find an adequate range of initial values on them, to be established previously to that identification method.

\section{Materials and methods}

This work is done over a solenoid common-rail type injector, model: DENSO 5031 06E18602 13H50A. A scheme is provided in Fig. 1. Injector operation is as follows: once the current is applied to the solenoid, the armature lifts, making the fuel to flow towards de control chamber and decreasing the pressure at the top of the command piston so that the needle rises, which starts the fuel injection into the combustion chamber. When the current finishes, the armature returns to its initial position pushing the needle down and stopping the fuel injection.

The accurate measurements related to each component of the injector (geometries, masses, physical properties, etc.) is a critical factor because the small dimensions of the ducts and elements require specific methodologies. In the present work, several methodologies are applied in order to obtain most of the characteristics of the injector under study [5] Fig. 2 shows an example of how the dimensions of the nozzle outlet orifices have been measured following the silicone methodology developed by previous authors [6]. This methodology allows quantifying the internal geometry of the injector.

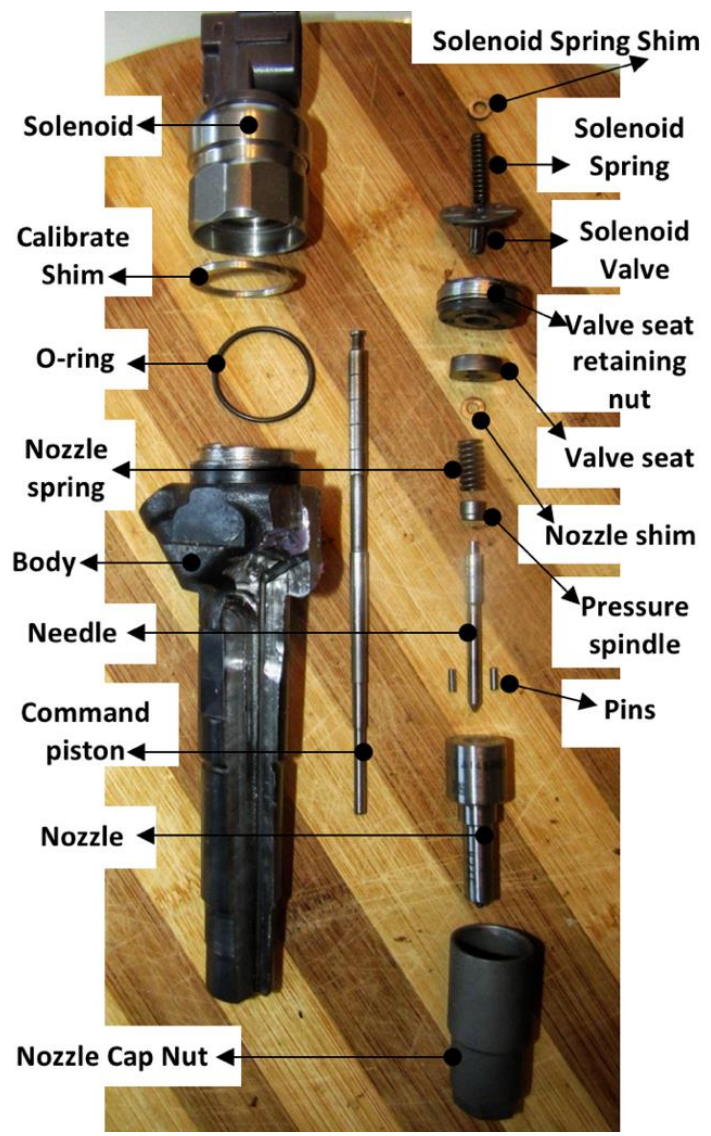

Fig. 1. Components of the injector.
In the present study, a set of 12 operating conditions is evaluated in a systematic way. Each condition is characterized by two parameters: the rail pressure (bar) and the injector energization signal (A). These operating conditions illustrate a wide range of engine behavior in which this injector is mounted. These input data, as well as the injection rate, were experimentally determined in previous work [7]. Fig. 3 shows the input (injector energization signal) and output (fuel injection rate) profiles associated with one of those operating regimes tested.

A commercial software package [8] is used to build a mathematical model to determine the fuel injection characteristics of a common-rail solenoid injector. This software includes several sub-models to simulate the following elements: needle, nozzles, spring, valves, boundaries, volumes, orifices and tubes. Previous authors used this software to successfully simulate different types of injection systems, such as in-line systems [9] and common-rail systems [10].
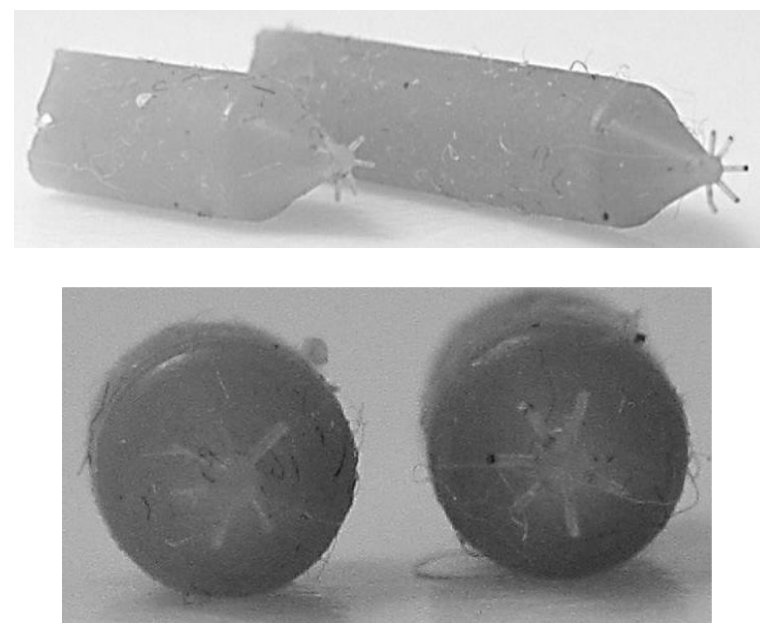

Fig. 2 Measurements examples of the internal geometry of a diesel nozzle using the silicone methodology

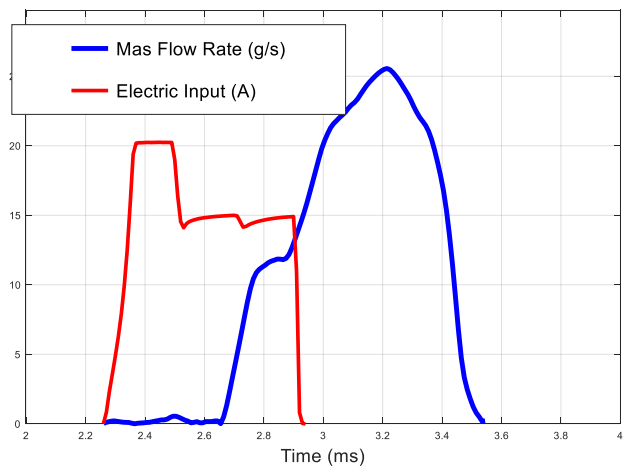

Fig. 3. Experimental measurements: injector energization signal injection and injection rate for the operating condition $60 \mathrm{Nm}$ and $1700 \mathrm{rpm}$ engine speed (rail pressure $=65 \mathrm{MPa}$ )

Model accuracy is determined by comparing its outputs with the experimental results. For this purpose, an objective function $\Psi$ has been defined to compare the modeled and the measured injection rates for the 12 operating conditions tested according to the recommendations for an appropriate design of 
experiments [11]. An individual objective function $\psi_{j}$ for each condition ' $j$ ' is evaluated by means of the following equation:

$$
\Psi_{j}=\left(\frac{1}{\tau_{j}}\right) \int_{0}^{\tau_{j}}\left[Y^{\prime}(\tau)-Y(\tau)\right]^{2} d \tau
$$

where $\tau_{j}$ refers to the injection duration time, $Y$ corresponds to the instantaneous modeled fuel injection rate along the time, and $Y^{\prime}$ is the corresponding measured injection rate.

The overall value $\Psi$ is obtained by adding all $\Psi_{j}(\mathrm{j}=1,2$, $\ldots, 12)$. It is important to note that, for any of those conditions, component characteristics maintain the same value. Although the initial measurement procedure provides most of the input data required by the software, there are 30 of these parameters whose values are not known with accuracy or they are unknown, such as amount stiffness and damping coefficients associated with different elements, etc. Therefore, they must be identified by an optimization algorithm.

Fig. 4 summarizes the identification procedure applied in this work. Each step starts assigning a certain value for each unknown parameter. Then, for each condition, the experimental input signals (in this case, the injector energization) are used to simulate several injector output parameters. Among them, the fuel injection rate is used in the present work to calculate $\Psi_{j}$ and, subsequently, an overall $\Psi$.

This estimated value and its variation along the procedure makes the input set of unknown parameters to be changed until a minimum is reached.

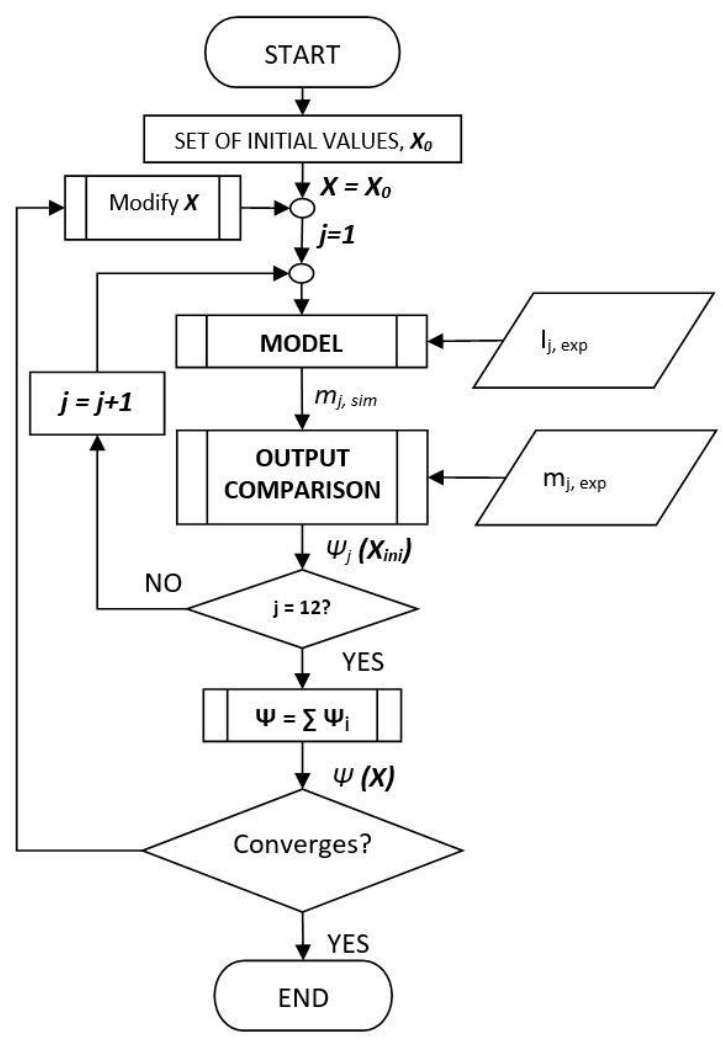

Fig. 4 Identification procedure scheme.
Two optimization methods have been used. The first one is the Non-Linear Programming by Quadratic Lagrangian method (NLPQL) [1], [12]. It consists of a gradient-based local optimization method, which solves problems with smooth continuously differentiable objective functions and constraints. It requires an adequate starting point close to the global minimum or needs the merit function to be convex. The second optimization method is a GA procedure [2], [12] which is able to find solutions for nondifferentiable objective functions (or quite complex differentiable).

Previously to the application of the identification procedure, an initial set of values for those parameters must be established, as well as the searching range for each of them. This selection of values is not easy, because there are interactions between parameters that affect the outputs.

Sometimes, experience provides enough knowledge to approximate the value of some parameters, which allows us to take it as the center of its searching range and to narrow this range of variation. But in other cases, there is no information, thus a first approach must adopt a value for each parameter, where a global $\Psi$ is reached, and establish it as the center value of the range. The range can be selected by setting an interval calculated as a percentage of the central value. Since no information is available, it is difficult to establish those ranges.

Before applying the optimization procedure, it is needed a sensitivity assessment in order to decrease the number of parameters to be identified [4]: this sensitivity analysis evaluates the influence of the variation of each unknown parameter $\omega_{\mathrm{i}}$ on the model response $(\Psi)$ using a sensitivity coefficient $\left(S_{\mathrm{i}}\right)$ :

$$
S_{i}=\frac{\partial \psi}{\partial \omega_{i}}
$$

Once the assessment is done, the results are analyzed. Those parameters with very low influence on the simulated outputs can be removed from the identification procedure and the central values can be fixed. For those cases with acceptable sensitivity, the ranges can be maintained as they were established initially. On the contrary, in those cases with excessive variations, the initial range must be reduced so that a congruous change in the output parameter is obtained. This last analysis requires a deeper study focused on the injector component associated with this unknown parameter.

\section{Results}

First, based on our previous experience, a given random range is established for each parameter. Then, the sensitivity analysis is done, which provides the results shown in Fig. 5.

Since several parameters vary slightly (less than 10\%), they are removed from the identification process, and their centered values are fixed. Their parameters mainly correspond to characteristics associated to the needle and 
the piston, such as: preload, damping, and stiffness of needle spring; friction force in the needle; stop damping and stiffness in both needle and piston. It means that the behavior of these components can be reproduced with enough accuracy from their corresponding initial values, due to they provide very low variation in the simulated outputs even when they vary significantly.

In addition, other parameters show coherent relative variations. It implies that the adopted range can be assumed as appropriate for the identification process.

These parameters are: inlet throttle area, outlet throttle area, needle seat diameter and nozzle sac diameter. Additionally, four characteristics related to the armature present rational sensitivities as well.

Finally, the rest of the parameters selected for the sensitivity analysis show high variability. These parameters are the most important for the identification model. Apart from seven parameters associated with the armature, the minimum inlet throttle area also produces a high variability.

Evaluating the whole set of parameters, it is important to highlight: first, that the value of this last geometric value (inlet throttle area) must be carefully chosen before starting the identification process. The second task is the high uncertainty that the armature of the solenoid has in the injection model, while several terms present excessive variations.

Next, the identification process was carried out in order to evaluate results for these initial uncertainties, mainly due to the armature behavior and the inner correlation among parameters. Since the searching range of values is high, the internal algorithm has problems finding the direction in which the parameters vary along with the procedure. The result obtained for the simulated fuel injection rate is shown in Fig. 6.

The presented condition shows a satisfactory fitting, but there are some of the others that do not reach enough accuracy. The best results are reached for medium and high speeds at medium load. Higher discrepancies appear at extreme speeds and loads.

The number of parameters, the uncertainties associated to the armature modeling, as well as the complexity of the physics involved in the whole injection process, make difficult to obtain an accurate approximation.

If the identification process is performed for each operating condition, without considering the rest of them at the same time, each one may provide different final values for the same parameter, which makes no sense. For this reason, the procedure has been developed by batch, using the same value of each parameter for all the 12 conditions at each step of the optimization algorithm. It implies that some of the final curves do not match so well as they could. In this case, it seems that a local minimum is reached.
Regarding the second optimization method, i.e. via GA, the results are similar to those obtained from the first procedure, as well as the discrepancies. Thus, it implies that the dissimilarities found cannot be exclusively associated with the optimization algorithm used but also to the selected search range used to find the value of a parameter.

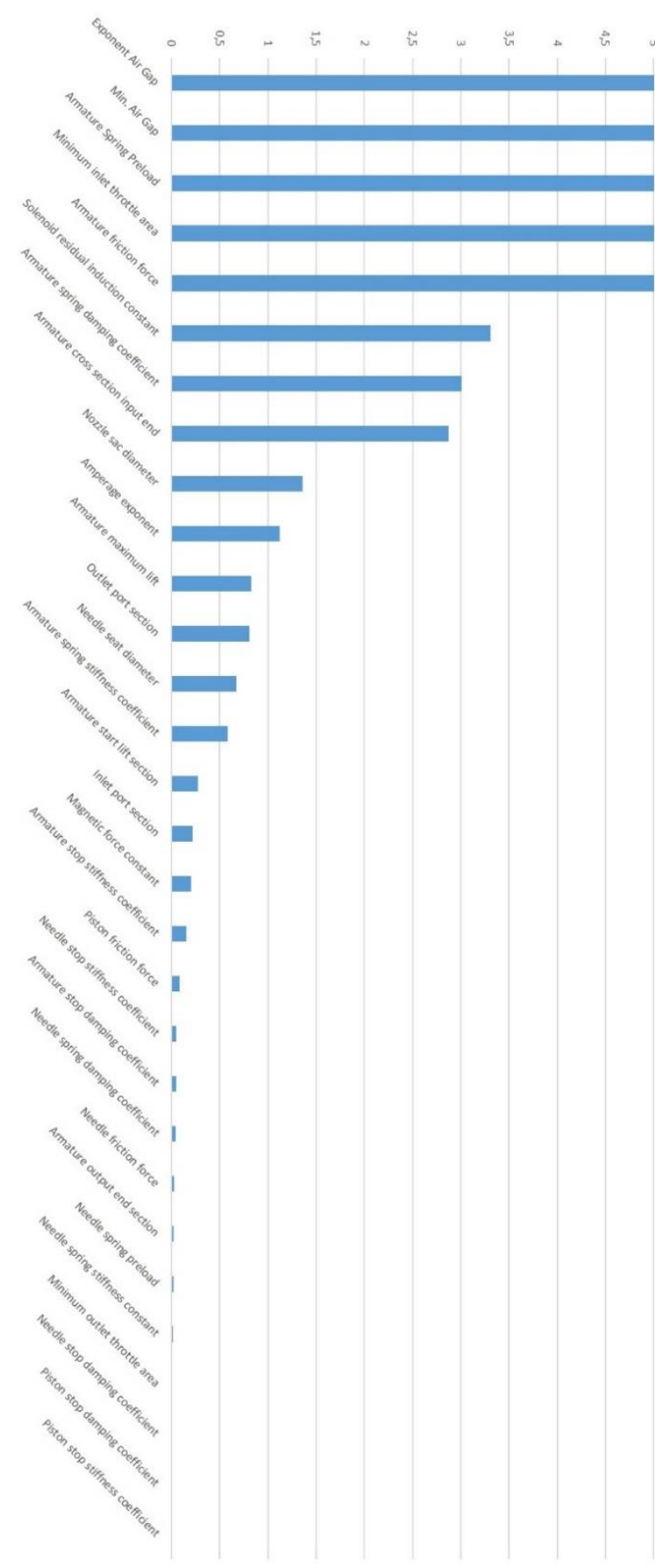

Fig. 5 Sensitivity results from an initial random range.

Further effort is needed to accurately determine those parameters with excessive sensitivity, by readapting the center value and the searching range, in order to assure the consistency of results. Also, a detailed study dealing with the armature must be engaged. 


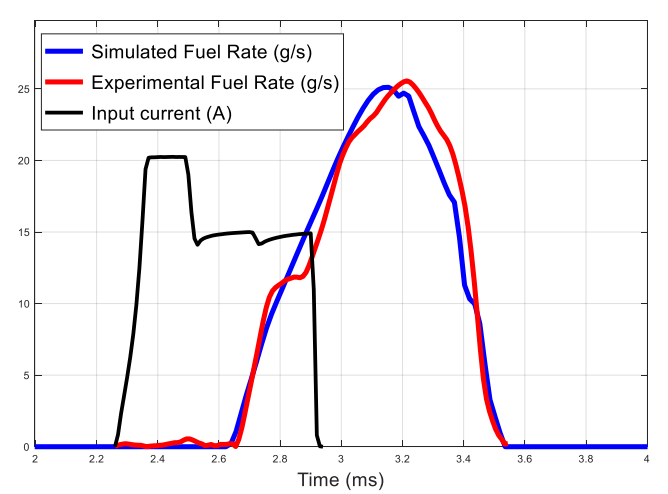

Fig. 6. Final results provided by the identification algorithm considering the operating condition selected in Fig. 3

\section{Conclusions}

The main conclusions of the present work are summarized as follows:

- Previously to the application of the identification procedure, it is important to perform an adequate selection of the initial centred values and the searching ranges. The interest of this work lies in the fact that it helps to fix the appropriated search range for determining the value of each parameter.

- The sensitivity assessment allows decreasing the number of significant parameters in the model.

- Several terms can be removed from the identification procedure, due to their low sensitivity. It implies that the number of terms to be identified can be reduced from 30 to 18 .

- On the other hand, some parameters need a deeper study, mainly those associated with the injector armature.

\section{Acknowledgement}

This work was supported by the Spanish Ministry of Science, Innovation and Universities [RTI2018-095923-BC21]. The usage of the AVL Boost ${ }^{\mathrm{TM}}$ Hydsim software in the framework of the University Partnership Program of AVL List GmbH is greatly acknowledged. Authors are also grateful for the provision of a paid internship grant to J.L. Perona-Navarro from the University of Jaén (nr. 173832).

\section{References}

[1] P.E. Gill, W. Murray, M. H. Wright, Practical Optimization, Academic Press, London (1981)

[2] D.T. Pham, D. Karaboga, Intelligent optimisation techniques, Springer (2000).

[3] C. Stürzebecher, J. Reß, C. Bohn, F. Märzke, R. Frase, “A diesel engine model including exhaust flap, intake throttle, LP-EGR and VGT. Part II: identification and validation", IFAC-PapersOnLine (2015), Vol. 48(15), pp. 60-65

[4] C.W.U. Wu, M. Hamada, Experiments: planning, analysis, and parameter design optimization, John Wiley \& Sons, Inc., New York (2000), pp. 205-255.

[5] L. Robles-Lorite, Estudio y caracterización de un inyector common-rail, Diploma work, University of Jaén (2019).
[6] V. Macian, V. Bermúdez, R. Payri, J. Gimeno, "New technique for determination of internal geometry of a diesel nozzle with the use of silicone methodology", Experimental Techniques (2003), Vol. March/April, pp. 39-43.

[7] J.A. Soriano, C. Mata, O. Armas, C. Ávila, "A zerodimensional model to simulate injection rate from first generation common rail diesel injectors under thermodynamic diagnosis", Energy (2018), Vol. 158, pp. 845-858.

[8] AVL BOOST, Hydsim Users Guide, AVL List Gmbh (2009).

[9] E. Torres-Jimenez, R. Dorado, B. Kegl, M. Kegl, "Onedimensional modeling and simulation of injection processes of bioethanol-biodiesel and bioethanol-diesel fuel blends", Fuel (2018), Vol. 227, pp. 334-344.

[10] P. Punov, "Research the fuel injection characteristics of a common-rail solenoid injector", Proceedings of BulTrans2015 (2015), pp. 153-158.

[11] E. Torres-Jiménez, O. Armas, L. Lesnik, F. Cruz-Peragón, "Methodology to simulate normalized testing cycles for engines and vehicles via design of experiments with low number of runs", Energy Conversion \& Management (2018), Vol. 177, pp. 817-832.

[12] AVL WORKSPACE DoE and Optimization, AVL List Gmbh, 2009. 